\title{
基于药效团的三维数据库搜索*
}

\author{
彭 涛周家驹 \\ (中国科学院过程工程研究所, 北京 100080)
}

\begin{abstract}
摘要 用表皮生长因子受体酪氨酸激酶抑制剂的药效团作为提问结构在三维数据库中进行了搜索. 从得到 的命中结构中挑选了 12 个化合物用柔性受体模型方法对其活性进行了预测，发现有 2 个化合物具有一定的 预测活性. 这 2 个化合物可能具有酪氨酸激酶抑制剂的活性, 可能作为先导化合物进行结构优化.
\end{abstract}

关键词: 酪氨酸激酶抑制剂, 药效团, 三维数据库搜索 中图分类号: O641

酪氨酸激酶在一系列细胞活动的信号传导途径 中起着重要的作用. 很多类型的肿瘤因为有功能紊 乱的生长因子受体酪氨酸激酶，从而导致不正确的 有丝分裂信号．因此在包括癌症和其他许多疾病的 治疗研究中, 酪氨酸激酶都是研究的目标和对象 ${ }^{[1]}$. 大约十年以前，很多药物公司刚刚开始研究信号传 导的时候，表皮生长因子受体 (EGFR) 酪氨酸激酶 是文献中最早描述的一个酪氨酸激酶，因此也成为 了药物发现的靶点. 在为数众多、结构各异的酪氨 酸激酶抑制剂中，有一类引起了人们特别的研究兴 趣, 那就是和 ATP 竞争与激酶催化区域结合的化合 物, 人们公认激酶的 ATP 作用位点是药物设计中引 人注目的研究对象 ${ }^{[2-5]}$.

三维结构搜索方法是一种最大限度地利用已有 分子的三维结构信息进行药物分子设计的方法. 由 于含有指定药效团的分子可能具有相应的生物活 性, 因此, 三维结构搜索方法可以在实验篮选之前为 数据库中分子的生物活性做预测. 三维结构搜索的 重要意义在于，当搜索结果中有一些结构全新的分 子时, 意味着可能有先导化合物的发现. 另外, 三维 结构搜索方法还具有如下的优点: 由于数据库中的 分子是已经存在的化合物，其合成方法是已知的甚 至已经成为商品, 可以直接购买, 从而可以较快地进 人生物测试阶段, 提高新药开发的效率. 因此, 三维 结构搜索是一种利用三维结构数据库来发现先导 化合物的快捷而有效的途径。近几年来, 三维结构

搜索已经成为药物开发研究中的一种重要辅助手 段 ${ }^{[6-7]}$.

我们用柔性受体模型 (FLARM $)^{[8]}$ 方法对一系 列的表皮生长因子受体酪氨酸激酶抑制剂进行了 $3 \mathrm{D}$ QSAR 和受体模型研究, 得到的活性位点和 Novartis 的药效团模型 ${ }^{[2]}$ 比较类似，并据此分析和 归纳出了表皮生长因子受体酪氨酸激酶抑制剂的 药效团 ${ }^{[9-11]}$. 我们利用这个药效团作为提问结构在 三维数据库中进行搜索, 以期得到新的先导化合 物.

\section{1 方 法}

表皮生长因子受体酪氨酸激酶抑制剂的药效 团如图 1 所示. 三维数据库搜索采用本实验室开

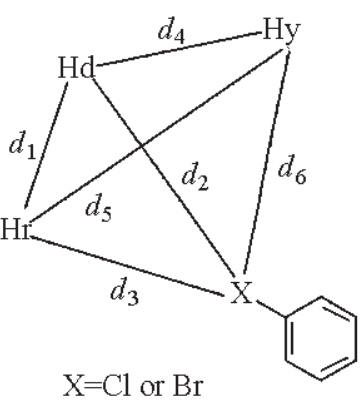

$d_{1}=(0.36 \pm 0.10) \mathrm{nm}$

$d_{2}=(0.92 \pm 0.10) \mathrm{nm}$

$d_{3}=(0.80 \pm 0.10) \mathrm{nm}$

$d_{4}=(0.48 \pm 0.10) \mathrm{nm}$

$d_{5}=(0.60 \pm 0.10) \mathrm{nm}$

$d_{6}=(0.95 \pm 0.10) \mathrm{nm}$

图 1 表皮生长因子受体酷氨酸激酶抑制剂的药效团

Fig. 1 The pharmacophore of epidermal growth factor receptor(EGFR) tyrosine kinase inhibitors

2003-02-18 收到初稿，2003-05-09 收到修改稿。

联系人: 周家驹 (E-mail: jjzhou@ lcc. icm. ac. cn; Tel: 010-62626703).

*国家基础 研究发展规划项目资助 (G1998051115) 
表 1 三维数据库搜索的结果

Table 1 Results of 3D database searching

\begin{tabular}{|c|c|c|c|c|}
\hline Database & Number of hits by & $t$ & Number of hits by & $t$ \\
\hline & $3 \mathrm{D}$ rigid search & $\min$ & 3D flexible search & $\min$ \\
\hline NCI-3D & 20 & 3.5 & 105 & 122 \\
\hline Cancer-3D & 7 & 3.0 & 184 & 94 \\
\hline AIDS-3D & 7 & 4. 0 & 224 & 111 \\
\hline
\end{tabular}

发的三维柔性搜索系统 $3 \mathrm{DFS}^{[12]}$ 进行, 三维数据库 为 NCI-3D、Cancer-3D 和 AIDS-3D ${ }^{[13]}$.

\section{2 结果和讨论}

三维数据库搜索的结果如表 1 所示. 从命中 结构中选取了 12 个可能具有酪氨酸激酶抑制剂活 性的结构, 选取的依据主要是药效团中四个组成部 分之间的相对位置关系，在本体系中由于角度的 限制比较宽松，因此目前药效团中没有包含角度因 素, 这一部分的判断是通过观察来进行的. 把这 12 个化合物和进行 $3 \mathrm{D}$ QSAR 以及受体模型研究的模 板分子(如图 2 所示)进行叠加. 在 3DFS 的搜索结 果中, 包含着每一个搜索命中结构中和药效团各部 分相对应的原子序号，按照这种对应关系把各个原 子和模板分子的相应原子进行叠加. 用 FLARM 方 法对其生物活性进行预测。其预测结果如表 2 所 示.

表 2 中列出了 FLARM 构建的前 10 位受体模 型对这 12 个化合物活性预测结果中的最低值和最 高值. 这个预测结果和 FLARM 方法的特点有着直 接的关系. FLARM 方法是基于 粗互作用越强, 生 物活性越高” 的假设, 因此和传统的 3D QSAR 方法 不同，FLARM 方法可以预测和训练集结构差异很 大的化合物的生物活性，挑出结构类似物的框架. 在表 2 中，某些化合物的活性预测值很低，这是由 于这些化合物和受体之间的作用能量太高，原因可 能是部分原子发生冲突导致能量急剧升高。在

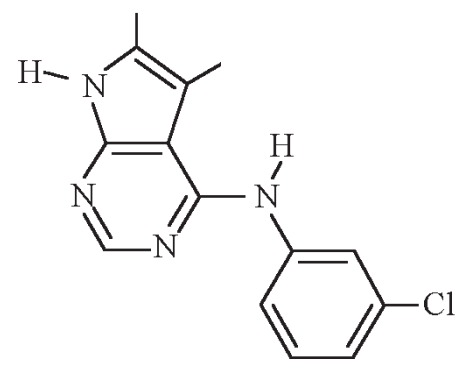

图 23 QSAR 研究中的模板分子

Fig. 2 The template molecule in 3D QSAR research
表 2 命中结构生物活性的预测结果

Table 2 The prediction results of the hits'biological activities

\begin{tabular}{crr}
\hline \multirow{2}{*}{$\begin{array}{c}\text { Compound } \\
\text { number }\end{array}$} & \multicolumn{2}{c}{$p K \mathrm{IC}_{50}$} \\
\cline { 2 - 3 } $\mathbf{1}$ & 2.04 & \multicolumn{1}{c}{ Max } \\
\hline $\mathbf{2}$ & -8.01 & 3.48 \\
$\mathbf{3}$ & 3.83 & -7.63 \\
$\mathbf{4}$ & 3.77 & 5.07 \\
$\mathbf{5}$ & 5.91 & 4.82 \\
$\mathbf{6}$ & 1.69 & 7.43 \\
$\mathbf{7}$ & 2.59 & 3.85 \\
$\mathbf{8}$ & -8.43 & 4.26 \\
$\mathbf{9}$ & 3.83 & -6.76 \\
$\mathbf{1 0}$ & -7.33 & 5.00 \\
$\mathbf{1 1}$ & 3.03 & -5.52 \\
$\mathbf{1 2}$ & 6.10 & 4.86 \\
& & 7.32 \\
\hline
\end{tabular}

FLARM 方法目前的版本中, 叠加结果的好坏对计 算结果还是有一定程度的影响. 在 FLARM 方法的 下一个版本中，将把受体和配体分子进行对接，这 样即使化合物叠加得不是很好, 程序也会自动地调 整化合物和受体之间的相对位置, 从而把化合物的 结构类似程度和化合物的叠加对计算结果的影响 程度降低到最小. 当然, 计算量和计算时间会大大 增加。

从表 2 看出，全部 10 个受体模型对化合物 $\mathbf{5}$ 和 12 的活性预测值偏差较小, 活性预测值也比较 高, 因此这两个化合物有可能具有酪氨酸激酶抑 制剂的活性，当然是否有活性还需生物活性测定
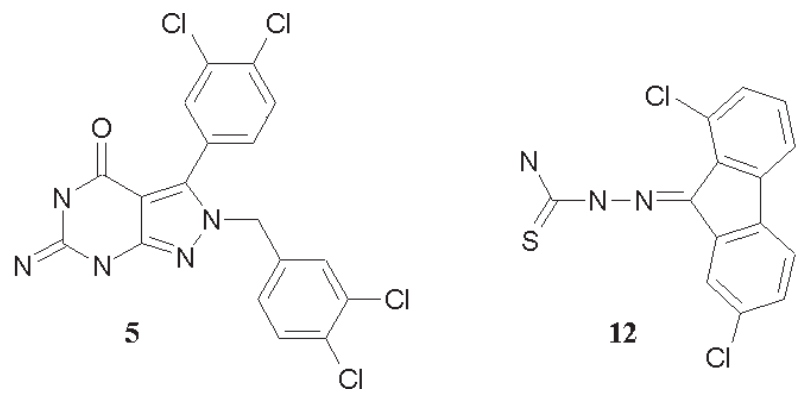

图 3 化合物 5 和 12 的结构

Fig. 3 The structures of compound 5 and 12 
和药理研究来证实. 化合物 $\mathbf{5}$ 和 12 的结构如图 3 所示. 可以看出, 化合物 $\mathbf{5}$ 和进行 $3 D$ QSAR 研究的 化合物结构差异不是很大，而化合物 12 则和原有 化合物结构差异很大，这说明进行三维数据库搜索 有可能发现结构全新的先导化合物.

\section{3 结 论}

用表皮生长因子受体酪氨酸激酶抑制剂的药 效团作为提问结构在三维数据库中进行了搜索. 从 得到的命中结构中挑选了 12 个化合物用 FLARM 方法对其生物活性进行了预测, 发现有 2 个化合物 具有一定的预测活性。这 2 个化合物可能具有酪氨 酸激酶抑制剂的活性，可能可以作为先导化合物进 行结构优化，当然是否有活性还需生物活性测定和 药理研究来证实.

\author{
References \\ $1 \quad$ Bishop, J. M. Science, 1987, 235: 305 \\ 2 Traxler, P. ; Furet, P. Pharmacol. Ther., 1999, 82: 195 \\ 3 Traxler, P. ; Furet, P. ; Mett, H. ; Buchdunger, E. ; Meyer, T. ;
}

Lydon, N. J. Med. Chem., 1996, 39: 2285

4 Traxler, P. ; Bold, G. ; Frei, J. ; Lang, M. ; Lydon, N. ; Mett, H. ; Buchdunger, E. ; Meyer, T. ; Mueller, M. ; Furet, P. J. Med. Chem., 1997, 40: 3601

5 Traxler, P. ; Green, J. ; Mett, H. ; Sequin, U. ; Furet, P. J. Med. Chem., 1999, 42: 1018

6 Martin, Y. C. J. Med. Chem., 1992, 35: 2145

7 Wang, S. M. ; Zaharevitz, D. W. ; Sharma, R. ; Marquez, V. E. ; Lewin, N. E. ; Du, L. ; Blumberg, P. M. ; Milne, G. W. A. J. Med. Chem., 1994, 37: 4479

8 Pei, J. F. ; Zhou, J. J. Acta Chimica Sinica, 2002, 60: 973 [裴 剑锋, 周家驹. 化学学报 (Huaxue Xиebao), 2002, 60: 973]

9 Peng, T. ; Pei, J. F. ; Zhou, J. J. J. Chem. Inf. Comput. Sci., 2003, 43: 298

10 Peng, T. ; Pei, J. F. ; Zhou, J. J. Acta Chimica Sinica, 2003, 61: 29 [彭 涛, 裴剑锋, 周家驹. 化学学报 (Huaxue Xuebao), 2003, 61: 29]

11 Peng, T. ; Pei, J. F. ; Zhou, J. J. Acta Chimica Sinica, 2003, 61: 430 [彭 涛, 裴剑锋, 周家驹. 化学学报 (Huaxue Xuebao), 2003, 61: 430]

12 Wang, T. ; Zhou, J. J. J. Mol. Model., 1999, 5: 231

13 Milne, G. W. A. ; Nicklaus, M. C. ; Wang, S. M. J. Chem. Inf. Comput. Sci., 1994, 34: 1219

\title{
Pharmacophore-based 3D Database Searching *
}

\author{
Peng Tao Zhou Jia-Ju
}

(Institute of Process Engineering, Chinese Academy of Sciences, Beijing 100080)

\begin{abstract}
A 3D database searching was conducted with 3DFS in 3D database using the pharmacophore of epidermal growth factor receptor(EGFR) tyrosine kinase inhibitors. Twelve compounds were selected from the hits and their biological activities were predicted using the Flexible Atom Receptor Model(FLARM) method. Two compounds among them were found to have high activities according to the prediction results. So these two compounds may have the inhibition function and structure optimization can be done with these two lead compounds.
\end{abstract}

Keywords: Tyrosine kinase inhibitor, Pharmacophore, 3D database searching 